\title{
Heterogeneidad ambiental y su relación con la diversidad y abundancia de la comunidad de peces en un sistema costero del Golfo de México
}

\author{
Arturo Aguirre-León ${ }^{1}$, Hilda Elín Pérez-Ponce ${ }^{2} \&$ Silvia Díaz-Ruiz $^{3}$ \\ 1. Laboratorio Ecología Costera y Pesquerías, Departamento El Hombre y su Ambiente, UAM-Xochimilco. A.P. 23-181, \\ C.P. 04960, D.F. México; agla1269@correo.xoc.uam.mx \\ 2. Comisión Nacional para el Conocimiento y Uso de la Biodiversidad (CONABIO). Liga Periférico- Insurgentes Sur \\ 4903, Parques del Pedregal, Tlalpan, C.P. 14010, D.F. México; hperez@conabio.gob.mx \\ 3. Laboratorio Ictiología y Ecología Costera, Departamento de Hidrobiología, UAM-Iztapalapa. A.P. 55-535, C.P. \\ 09340, D.F. México; sdr@xanum.uam.mx
}

Recibido 13-III-2013. Corregido 09-X-2013. Aceptado 07-XI-2013.

\begin{abstract}
Environmental heterogeneity and its relationship with diversity and abundance of the fish community in a coastal system of Gulf of Mexico. The coastal lagoons of Veracruz, Gulf of Mexico, include a great variety of biological resources. These resources, especially fish communities, have been barely described and that require more ecological studies. With this aim, this investigation analyzed the spatial and temporal variation of diversity, abundance and assemblages of the fish community and its relationship with physicalchemical parameters of the Chica-Grande coastal system. For this, eight defined sites were monthly sampled for water characteristics and fish community composition (10min hauls of $1500 \mathrm{~m}^{2}$ a shrimp trawl net), between September 2005 and November 2006. The spatial-temporal variation of physical-chemical parameters, allowed the definition of two contrasting environments according to salinity, temperature, transparency, dissolved oxygen and depth gradients. A total of 1947 fishes were collected for a total weight of $57.88 \mathrm{~kg}$. From these, 22 species, 20 genera and 14 families were identified; and four species were new records for the system. As it was detected for the physical-chemical conditions, the diversity of the fish community also showed a spatial gradient, with high values $\left(\mathrm{H}^{\prime}=2.37, \mathrm{D}=3.35, \mathrm{~J}^{\prime}=0.82\right)$ in the brackish habitat, and low ones in freshwater environments. Fish abundance did not show such a marked gradient response, however, it was higher in the freshwater habitat. The highest diversity $\left(\mathrm{H}^{\prime}=2.05\right)$ and species richness $(\mathrm{D}=2.99)$ was recorded during the North winds ("nortes") months (November-February), while density and biomass were higher $\left(0.034 \mathrm{ind} . / \mathrm{m}^{2}, 1.42 \mathrm{~g} / \mathrm{m}^{2}\right)$ during the rainy months (July-October). The Importance Value Index (IVI) defined six dominant species accounting for $77.8 \%$ of the fish abundance and $87.9 \%$ of total catch by weight. The Canonical Correspondence Analysis (CCA) showed that the fish-habitat relationship was explained by $68 \%$ of total variance for the two first axes, where salinity, transparency and temperature changes were the most important environmental variables in determining the fish community composition and distribution. In this study, spatial gradients of environmental variables explained the changes in fish species composition and abundance; while the temporal gradients, the fish community structure and its relationship with seasonal patterns of habitat use by the species during their life cycles. Rev. Biol. Trop. 62 (1): 145-163. Epub 2014 March 01.
\end{abstract}

Key words: environmental heterogeneity, community structure, fish-habitat relationships, Chica-Grande lagoon, Veracruz, México.

Las lagunas costeras son ecosistemas que se caracterizan por la mezcla de aguas continentales y marinas, con alta producción primaria y variedad de hábitats estuarinos. Presentan procesos ambientales y ecológicos complejos, resultado de marcadas variaciones ambientales espaciales y temporales causadas por la descarga estacional de los ríos o el intercambio mareal (Snedden, Cable \& Kjerfve, 2013). En estos sistemas se registra alta diversidad de especies que los utilizan como áreas de alimentación, crianza, reproducción 
y protección donde los peces constituyen el grupo más representativo por su riqueza, distribución y abundancia (Lara-Domínguez, Contreras-Espinosa, Castañeda-López, BarbaMacías \& Pérez-Hernández, 2011a). Los peces desarrollan interacciones complejas desde el mar hacia los sistemas lagunares, como reproducción, reclutamiento o alimentación, las cuales se relacionan con los patrones de migración y el uso diferencial de los hábitats como respuesta a los cambios estacionales y espaciales de las variables ambientales (Gelwick, Akin, Arrington \& Winemiller, 2001; Barletta, Barletta-Bergan, Saint-Paul \& Hubol, 2005; Able, 2005; Miller \& Skilleter, 2006). Esto influye en la dinámica espacial y temporal de la diversidad y abundancia de las especies, así como en la composición de los ensambles de peces en el sistema (Pombo, Elliott \& Rebelo, 2005; Barreiros et al., 2009; ArceoCarranza, Vega-Cendejas, Montero-Muñoz \& Hernández, 2010).

El litoral de Veracruz, México, incluye al menos 18 lagunas costeras que cubren un área de $1166 \mathrm{~km}^{2}$, por lo que existe una amplia variedad de recursos biológicos ocupando el cuarto lugar en la producción pesquera nacional (Lara-Domínguez et al., 2011b). Destaca el sistema lagunar Chica-Grande por su riqueza de recursos pesqueros como mojarra (Eugerres plumieri), robalo (Centropomus parallelus) y tilapia (Oreochromis niloticus) para consumo local. No obstante, se requiere avanzar sobre el conocimiento de la composición de especies y la influencia de las variables ambientales en los cambios espaciales y temporales de la estructura de la comunidad y los ensambles de peces. Algunos estudios en este sistema sólo incluyen listas de especies, como los realizados por Kobelkowsky (1991), Chávez, Rocha \& Ramírez (2005) y Arzola-Vargas \& Bautista-Chávez (2010). Considerando la importancia ecológica y económica de los peces como recursos y la poca información del sistema Chica-Grande, este estudio amplía la información ecológica sobre las comunidades de peces en lagunas costeras del Golfo de México de acuerdo con los siguientes objetivos: 1) analizar la dinámica fisicoquímica en escala espacial y temporal del sistema, 2) determinar los principales patrones de variación espacial y temporal de diversidad, abundancia y ensambles de peces con relación a las principales variables ambientales y 3) analizar el patrón de utilización del sistema por las especies dominantes.

\section{MATERIALES Y MÉTODOS}

Área de estudio: El sistema lagunar Chica-Grande se localiza en la parte occidental del Golfo de México (20 $02^{\prime}$ y $20^{\circ} 06^{\prime} \mathrm{N}-96^{\circ} 38^{\prime}$ y $96^{\circ} 41^{\prime} \mathrm{W}$ ) en el Estado de Veracruz. Tiene una superficie total de $22.5 \mathrm{~km}^{2}$ e incluye dos lagunas asociadas (Fig. 1). La primera llamada Laguna Chica (LC) al norte con $3 \mathrm{~km}$ de longitud y $0.8 \mathrm{~km}$ de ancho y la segunda, Laguna Grande (LG) al sur con una longitud de $4.7 \mathrm{~km}$ y $1.5 \mathrm{~km}$ de ancho, ambas están comunicadas por un canal estrecho llamado el Caño. Es un sistema somero, con una profundidad media de $0.9 \mathrm{~m}$ y amplitud de marea de $0.4 \mathrm{a}$ $0.6 \mathrm{~m}$. Tiene influencia marina a través de una boca de conexión de $300 \mathrm{~m}$ de anchura que se conecta por un canal sinuoso de aproximadamente $3.5 \mathrm{~km}$ de largo localizado al sureste de LG. Recibe la descarga de varios ríos de poco caudal: Cerritos, Diamante y Carey (Fig. 1). El clima es cálido subhúmedo con lluvias en verano (García, 2004). Se reconocen tres épocas climáticas: secas (marzo a junio), lluvias (julio a octubre) y nortes (noviembre a febrero) (Utrera-López \& Moreno-Casasola, 2008). Existen diferentes ambientes acuáticos en el sistema como vegetación circundante de manglar en áreas salobres y de baja salinidad, vegetación emergente y sumergida, bancos de macroalgas rojas (Gracillaria sp.) en el sur de la laguna y áreas de alta sedimentación (Moreno-Casasola et al., 2010; Lara-Domínguez et al., 2011a).

Actividades de campo y laboratorio: La recolección de peces se llevó a cabo en ocho estaciones de muestreo durante nueve meses, entre septiembre 2005 a noviembre 2006 (Fig. 1). Las estaciones se establecieron 


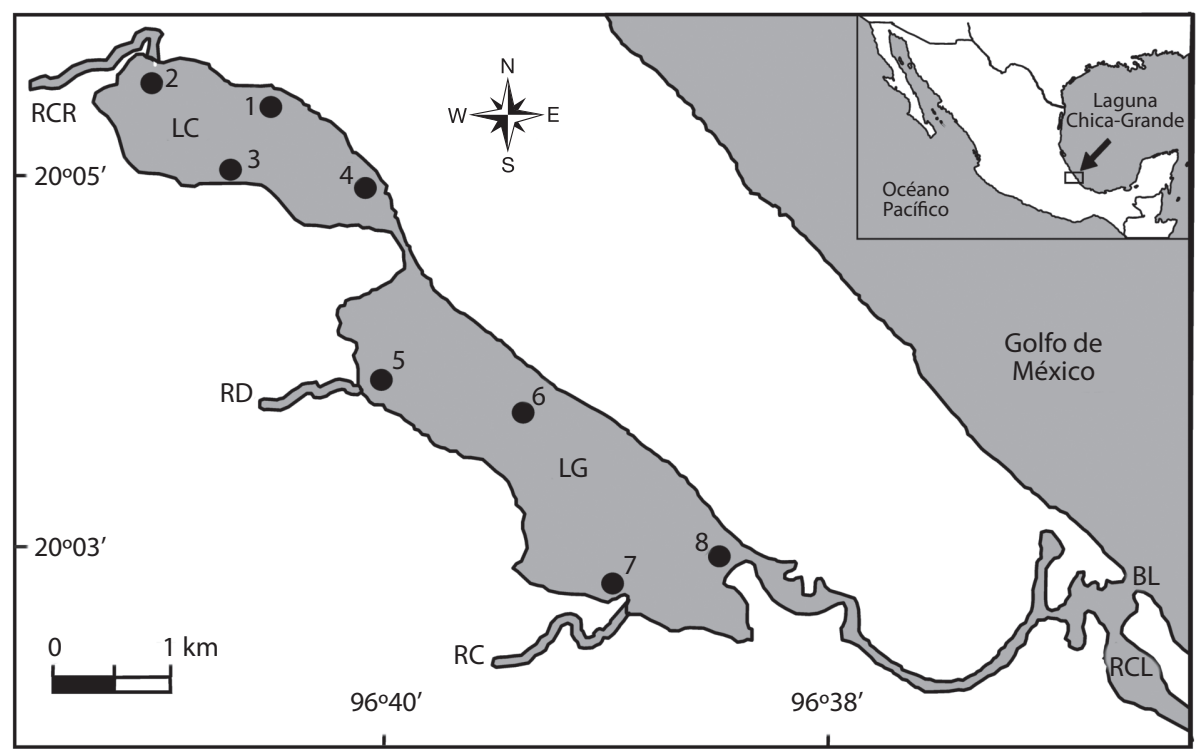

Fig. 1. Sistema lagunar Chica-Grande, Veracruz. Estaciones de muestreo $(\bullet)$, principales características fisiográficas del área y ríos que drenan al sistema. Laguna Chica (LC), Laguna Grande (LG), Boca Lagunar (BL), Río Colipa (RCL), Río Carey (RC), Río Diamante (RD), Río Cerritos (RCR).

Fig. 1. Chica-Grande lagoon system, Veracruz. The location of sampling stations $(\bullet)$, the main physiographic features of the study area and rivers draining into the system are noted. Chica Lagoon (LC), Grande Lagoon (LG), Lagoon Mouth (BL), Colipa River (RCL), Carey River (RC), Diamante River (RD), Cerritos River (RCR).

considerando áreas de influencia fluvial y marina, vegetación emergente y sumergida. Las capturas mensuales fueron diurnas, y en cada estación se realizó un lance de $10 \mathrm{~min}$ por día, con un área de arrastre de $1500 \mathrm{~m}^{2}$; en general, se realizó un total de 72 lances durante el estudio. Para esto, se utilizó una red de arrastre camaronera de $5.0 \mathrm{~m}$ de largo, $2.5 \mathrm{~m}$ de abertura de trabajo y luz de malla de $1.9 \mathrm{~cm}$. Las muestras fueron fijadas en formaldehído al $4 \%$ neutralizado con borato de sodio, y posteriormente preservadas en etanol al $70 \%$ (Díaz-Ruiz, Aguirre-León, Calva-Benítez \& Barba-Macías, 2012). En cada muestreo se registró la salinidad, temperatura y oxígeno disuelto con un multianalizador YSI-3800; la profundidad con una sonda graduada $(\mathrm{cm})$ y la transparencia con un disco de Secchi $(\mathrm{cm})$. Se realizaron anotaciones sobre la presencia de vegetación circundante y sumergida. En el laboratorio se midió longitud total (LT) de los peces con un ictiómetro graduado en centímetros y se pesaron con una balanza digital (precisión 0.01g). La identificación taxonómica de los peces se realizó con claves del Golfo de México (Cervigón, 1991-1994; Fischer, 1995; Castro-Aguirre, Espinosa-Pérez \& Schmitter-Soto, 1999; Froese \& Pauly, 2012).

Se calcularon los parámetros ecológicos de la comunidad: diversidad $\mathrm{H}^{\prime}$ (Shannon \& Weaver, 1963), riqueza de especies D (Margalef, 1969) y equitatividad J' (Pielou, 1966), así como la densidad (ind. $\left./ \mathrm{m}^{2}\right)$, biomasa $\left(\mathrm{g} / \mathrm{m}^{2}\right)$ y peso promedio de los peces (g/ind). La dominancia de las especies se determinó mediante el Índice de Valor de Importancia (IVI) como la suma del porcentaje en número y peso de las especies y la frecuencia de aparición (De la Cruz-Agüero, 1994). Para estimar diferencias estadísticas espaciales (entre estaciones y lagunas) y temporales (entre meses y épocas climáticas del año) de las variables ambientales y parámetros ecológicos de la comunidad, fue evaluada previamente la homogeneidad 
de varianzas entre grupos utilizando la prueba de Levene (1960). En los casos necesarios se hicieron transformaciones logarítmicas. Cuando se cumplió la homogeneidad de varianzas se utilizó una prueba ANOVA-F-, cuando no se cumplió fue utilizada la prueba KruskalWallis-H- (Zar, 1999). Para todas las pruebas anteriores se consideró un nivel de significancia $\mathrm{p}<0.05$ (Zar, 1999; Hair, Anderson, Tatham \& Black, 2001). Se realizó análisis de agrupamiento "cluster" utilizando los parámetros fisicoquímicos para definir hábitats y los de diversidad para analizar afinidad entre ellos. La métrica utilizada fue la distancia euclidiana mediante ligamiento promedio ponderado (Hair et al., 2001). Se realizó un análisis de correspondencia canónica (ACC) para evaluar la relación entre las especies de peces y las variables ambientales del sistema (ter Braak \& Verdonshot, 1995). Para este fin, se utilizó una matriz de abundancia de las especies por estación y mes, y una matriz de datos fisicoquímicos. El ACC fue interpretado usando el programa Multivariate Statistical Package, versión 3.1.

\section{RESULTADOS}

Variación espacial y temporal de los parámetros ambientales: El análisis de los resultados fisicoquímicos mostró un evidente gradiente ambiental espacial con valores altos en la parte sur y bajos en la porción norte del sistema. La salinidad promedio más alta (16.7 \pm 3.2 ) se registró en la estación 8 (Fig. 2A) y la más baja $(9.4 \pm 4.4)$ en la estación 1. Espacialmente, sólo este parámetro mostró diferencias significativas entre sitios (ANOVA- $\mathrm{F}=7.05$, $\mathrm{p}<0.0001)$. La temperatura del agua presentó el valor promedio más alto $\left(28.6^{\circ} \mathrm{C} \pm 4.7\right)$ en la estación 7 (Fig. 2B) y el más bajo $\left(27^{\circ} \mathrm{C} \pm 4.3\right)$ en la estación 1. El oxígeno disuelto promedio más alto $(6.3 \mathrm{mg} / \mathrm{L} \pm 1.1)$ se registró en la estación 6 (Fig. 2C), mientras que el más bajo $(4.5 \mathrm{mg} / \mathrm{L} \pm 1.6)$ en la estación 1 . La transparencia promedio más alta $(29.7 \mathrm{~cm} \pm 10.7)$ se presentó en la estación 6 (Fig. 2D) y la más baja
$(25.6 \mathrm{~cm} \pm 10.4)$ en la estación 2. La profundidad del sistema presentó valores promedios mayores $(0.97 \mathrm{~m})$ en las estaciones $1 \pm 0.11$ y $2 \pm 0.14$ (Fig. 2E) y menores $(0.76 \mathrm{~m})$ en las estaciones $7 \pm 0.17$ y $8 \pm 0.2$.

En contraste para la escala temporal, todas las variables ambientales mostraron cambios marcados con diferencias estadísticas significativas. La salinidad promedio más alta (18.2 \pm 2.6 ) se presentó en junio (Fig. 2F), mientras que la más baja $(8.0 \pm 2.2)$ en febrero (Kruskal Wallis- $\mathrm{H}=35.57, \mathrm{p}<0.0001)$. La temperatura promedio más alta $\left(32.6^{\circ} \mathrm{C} \pm 0.8\right)$ se registró en junio (Fig.2G) y la más baja $\left(20.5^{\circ} \mathrm{C} \pm 0.4\right)$ en diciembre como reflejo de la variación térmica regional (Kruskal Wallis-H=67.13, $\mathrm{p}<0.0001$ ). El oxígeno disuelto promedio fue mayor (8.8mg/L \pm 0.7 ) en diciembre (Fig. $2 \mathrm{H}$ ), y menor $(3.8 \mathrm{mg} / \mathrm{L} \pm 0.4)$ en marzo (ANOVA-F=15.69, $\mathrm{p}<0.0001)$. La transparencia presentó su valor promedio más alto $(51.5 \mathrm{~cm} \pm 6.4)$ en septiembre (Fig. 2I), y el más bajo $(17.9 \mathrm{~cm} \pm 4.2)$ en octubre (ANOVA-F=9.04, $\mathrm{p}<0.0001$ ). La profundidad promedio del sistema varió de $0.73 \mathrm{~m} \pm 0.13$ en junio (Fig. 2J) a $1.19 \mathrm{~m} \pm 0.19$ en septiembre (ANOVA-F $=8.15, \mathrm{p}<0.0001$ ). Los valores promedio de los parámetros fisicoquímicos entre épocas del año se muestran en el cuadro 1. El análisis de varianza indicó diferencias significativas entre estas.

Con base en la variación espacio-temporal de los parámetros físicoquímicos, el análisis clúster (Fig. 3A) mostró dos grupos: el primero integrado por las estaciones 1 a 4 que corresponden a Laguna Chica (LC) y el segundo formado por las estaciones 5 a 8 presentes en Laguna Grande (LG). Los parámetros ambientales que definieron estos grupos o ambientes fueron la salinidad y el oxígeno, ya que el análisis de varianza indicó diferencias significativas entre LC y LG (Cuadro 2). De acuerdo con este cuadro, LC se caracterizó por ser una zona más profunda con menor salinidad, oxígeno disuelto y transparencia, mientras que LG se distinguió por ser una zona más somera con mayor salinidad, oxígeno disuelto y transparencia. 

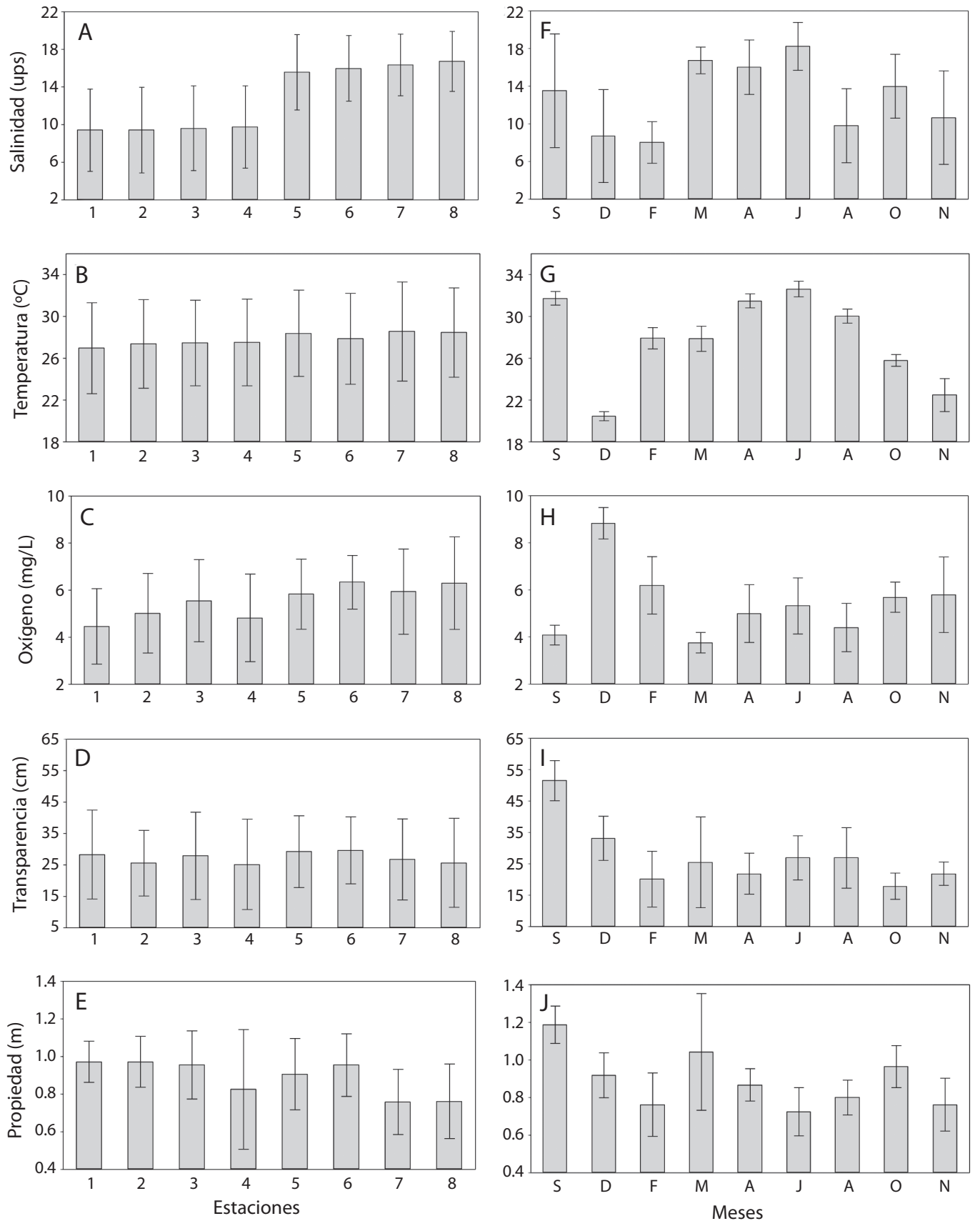

Fig. 2. Variación espacial y temporal de los parámetros fisicoquímicos del sistema Chica-Grande.

Fig. 2. Spatial and temporal variation in physico-chemical parameters in the Chica-Grande system. 
CUADRO 1

Valores promedio y estimadores estadísticos de los parámetros fisicoquímicos y parámetros comunitarios para cada época climática en el sistema Chica-Grande, Veracruz

TABLE 1

Average values and statistical estimators of the physico-chemical variables and community parameters for each lagoon in the Chica-Grande system, Veracruz

\begin{tabular}{|c|c|c|c|c|c|}
\hline \multirow{2}{*}{ Parámetro } & \multicolumn{3}{|c|}{ Épocas del año } & \multirow{2}{*}{ Estimador } & \multirow{2}{*}{$\mathrm{p}$} \\
\hline & Secas & Lluvias & Nortes & & \\
\hline Salinidad & 17.0 & 12.4 & 9.1 & $\mathrm{H}=29.4$ & $<0.0001^{*}$ \\
\hline Temperatura & 30.7 & 29.2 & 23.6 & $\mathrm{H}=35.8$ & $<0.0001^{*}$ \\
\hline Oxígeno disuelto & 4.8 & 4.8 & 6.9 & $\mathrm{H}=21.1$ & $<0.0001^{*}$ \\
\hline Transparencia & 24.7 & 32.1 & 25.0 & $\mathrm{H}=1.93$ & 0.380 \\
\hline $\mathrm{H}^{\prime}$ & 1.41 & 1.16 & 1.36 & $\mathrm{~F}=1.20$ & 0.307 \\
\hline $\mathrm{D}$ & 1.65 & 1.48 & 1.64 & $\mathrm{~F}=0.21$ & 0.810 \\
\hline$J^{\prime}$ & 0.76 & 0.73 & 0.69 & $\mathrm{~F}=0.53$ & 0.588 \\
\hline Ind. $/ \mathrm{m}^{2}$ & 0.021 & 0.012 & 0.022 & $\mathrm{~F}=2.36$ & 0.133 \\
\hline $\mathrm{g} / \mathrm{m}^{2}$ & 0.683 & 0.259 & 0.679 & $\mathrm{~F}=1.91$ & 0.155 \\
\hline g/ind. & 26.0 & 22.5 & 26.9 & $\mathrm{~F}=0.31$ & 0.733 \\
\hline
\end{tabular}

* Parámetros con diferencias significativas.

\section{CUADRO 2}

Valores promedio y estimadores estadísticos de los parámetros fisicoquímicos y parámetros comunitarios para cada laguna del sistema Chica-Grande, Veracruz

TABLE 2

Average values and statistical estimators of the physico-chemical variables and community parameters for each lagoon in the Chica-Grande system, Veracruz

\begin{tabular}{lcccc}
\multicolumn{1}{c}{ Parámetro } & Laguna Chica & Laguna Grande & Estimador & $\mathrm{p}$ \\
Salinidad & 9.55 & 16.15 & $\mathrm{H}=26.40$ & $<0.0001 *$ \\
Temperatura & 27.32 & 28.31 & $\mathrm{~F}=0.66$ & 0.417 \\
Oxígeno disuelto & 4.96 & 6.10 & $\mathrm{~F}=7.96$ & $0.006 *$ \\
Transparencia & 26.7 & 27.8 & $\mathrm{~F}=0.003$ & 0.954 \\
Profundidad & 0.93 & 0.85 & $\mathrm{~F}=3.28$ & 0.074 \\
$\mathrm{H}$ ' & 1.19 & 1.44 & $\mathrm{~F}=4.05$ & $0.048 *$ \\
$\mathrm{D}$ & 1.35 & 1.90 & $\mathrm{~F}=11.3$ & $0.001 *$ \\
$\mathrm{~J}$ ' & 0.70 & 0.77 & $\mathrm{~F}=1.19$ & 0.278 \\
Ind./m ${ }^{2}$ & 0.019 & 0.018 & $\mathrm{~F}=0.10$ & 0.744 \\
$\mathrm{~g} / \mathrm{m}^{2}$ & 0.68 & 0.41 & $\mathrm{~F}=0.89$ & 0.349 \\
$\mathrm{~g} /$ ind. & 26.0 & 25.6 & $\mathrm{~F}=0.01$ & 0.917 \\
\hline
\end{tabular}

* Parámetros con diferencias significativas.

Composición ictiofaunística: En el sistema Chica-Grande fueron recolectados un total de 1947 peces, pertenecientes a 6 órdenes, 14 familias, 20 géneros y 22 especies. El cuadro 3 muestra la lista de peces en orden de importancia (IVI). La familia Gerreidae fue la mejor representada con cuatro especies: $E$. plumieri, Diapterus rhombeus, D. auratus y Eucinostomus melanopterus. La presencia de especies en el sistema varió de $11(50 \%)$ en 

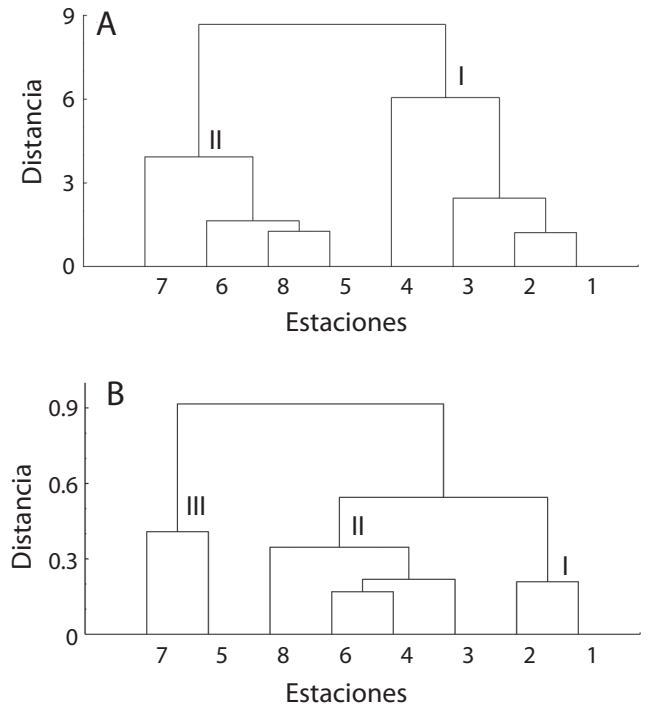

Fig. 3. Grupos de estaciones con características ambientales similares (A), y grupos de estaciones con valores similares de índices de diversidad H', D y J' para la comunidad de peces en el sistema Chica-Grande (B).

Fig. 3. Station groups with similar environmental characteristics (A), and station groups with similar values of the diversity indices H', D and J' for the fish community in the Chica-Grande system (B). septiembre, a 17 (77\%) en junio y octubre. Especies como Paraneetroplus fenestratus y Dormitator maculatus fueron registradas únicamente en LC, mientras que Archosargus probatocephalus, Eleotris amblyopsis, E. melanopterus, Opsanus beta y Symphurus plagiusa solo en LG. La especie exótica $O$. niloticus se capturó en ambas lagunas.

Variación espacial y temporal de la diversidad: $\mathrm{El}$ análisis espacial de H', D y J', mostró una tendencia similar al gradiente ambiental con los valores más altos en LG y los más bajos en LC (Fig. 4). De esta manera, el promedio más alto de H' $(1.64 \pm 0.35)$ se registró en la estación 5 (Fig. 4A), y para $\mathrm{D}(2.37 \pm 0.7)$ y J' $(0.86 \pm 0.1)$ en la estación 7 (Figs. 4 B y C); mientras que los más bajos para H' (1.03 \pm 0.7$), \mathrm{D}(1.2 \pm 0.8)$ y J' $(0.56 \pm 0.3)$ en la estación 1. Sólo el índice D presentó diferencias significativas entre los sitios $(\mathrm{F}=3.04$, $\mathrm{p}=0.008)$. Los valores promedio de diversidad entre LC y LG se muestran en el cuadro 2. El análisis de varianza mostró diferencias significativas para $\mathrm{H}^{\prime}$ y D $(\mathrm{p}<0.05)$.

CUADRO 3

Listado de las especies de peces colectadas en el sistema Chica-Grande, Veracruz

TABLE 3

List of the fish species collected in the Chica-Grande system, Veracruz

\begin{tabular}{|c|c|c|c|c|c|c|c|}
\hline Familia & Especies & $\mathrm{AB}$ & $\begin{array}{c}\text { Número } \\
(\%)\end{array}$ & $\begin{array}{l}\text { Peso } \\
(\%)\end{array}$ & $\begin{array}{c}\text { Frecuencia } \\
(\%)\end{array}$ & IVI & $\mathrm{CE}$ \\
\hline Centropomidae & Centropomus parallelus & $\mathrm{Cp}$ & 30.9 & 21.0 & 100.0 & 151.9 & EM \\
\hline Cichlidae & Oreochromis niloticus & On & 9.7 & 40.4 & 100.0 & 150.1 & $\mathrm{D}$ \\
\hline Gerreidae & Eugerres plumieri & Ep & 9.0 & 17.1 & 100.0 & 126.1 & EM \\
\hline Gerreidae & Diapterus rhombeus & $\mathrm{Dr}$ & 14.6 & 2.5 & 100.0 & 117.1 & EM \\
\hline Gerreidae & Diapterus auratus & $\mathrm{Da}$ & 10.3 & 1.6 & 100.0 & 111.9 & EM \\
\hline Ariidae & Cathorops melanopus & $\mathrm{Cm}$ & 3.2 & 5.4 & 100.0 & 108.6 & EM \\
\hline Achiridae & Achirus lineatus & $\mathrm{Al}$ & 6.2 & 0.5 & 88.9 & 95.5 & EM \\
\hline Paralichthyidae & Citharichthys spilopterus & $\mathrm{Cs}$ & 4.3 & 0.9 & 88.9 & 94.0 & EM \\
\hline Achiridae & Trinectes maculatus & $\mathrm{Tm}$ & 2.8 & 0.4 & 88.9 & 92.1 & EM \\
\hline Gobiidae & Gobionellus oceanicus & Go & 1.2 & 0.2 & 88.9 & 90.2 & ESM \\
\hline Batrachoididae & Opsanus beta $\mathbf{\square}$ & $\mathrm{Ob}$ & 1.4 & 3.3 & 77.8 & 82.5 & EM \\
\hline Centropomidae & Centropomus undecimalis & $\mathrm{Cu}$ & 0.6 & 2.2 & 77.8 & 80.5 & EM \\
\hline Eleotridae & Eleotris amblyopsis $\mathbf{\square}$ & $\mathrm{Ea}$ & 2.5 & 0.1 & 77.8 & 80.3 & $\mathrm{E}$ \\
\hline Mugilidae & Mugil curema & $\mathrm{Mc}$ & 1.7 & 3.4 & 66.7 & 71.7 & EM \\
\hline Eugraulidae & Anchoa mitchilli & Am & 0.5 & 0.0 & 66.7 & 67.2 & EM \\
\hline Sparidae & Archosargus probatocephalus $\mathbf{\square}$ & Ap & 0.3 & 0.7 & 44.4 & 45.4 & EM \\
\hline
\end{tabular}


CUADRO 3 (Continuación) / TABLE 3 (Continued)

\begin{tabular}{|c|c|c|c|c|c|c|c|}
\hline Familia & Especies & $\mathrm{AB}$ & $\begin{array}{c}\text { Número } \\
(\%)\end{array}$ & $\begin{array}{l}\text { Peso } \\
(\%)\end{array}$ & $\begin{array}{c}\text { Frecuencia } \\
(\%)\end{array}$ & IVI & $\mathrm{CE}$ \\
\hline Cichlidae & Paraneetroplus fenestrata $\bullet$ & $\mathrm{Pf}$ & 0.2 & 0.2 & 33.3 & 33.7 & $\mathrm{D}$ \\
\hline Carangidae & Hemicaranx amblyrhynchus & $\mathrm{Ha}$ & 0.3 & 0.1 & 22.2 & 22.6 & ESM \\
\hline Eleotridae & Gobiomorus dormitor & $\mathrm{Gd}$ & 0.1 & 0.2 & 22.2 & 22.6 & E \\
\hline Gerreidae & Eucinostomus melanopterus & Em & 0.2 & 0.0 & 22.2 & 22.4 & EM \\
\hline Eleotridae & Dormitator maculatus $\bullet$ & $\mathrm{Dm}$ & 0.1 & 0.0 & 11.1 & 11.2 & $\mathrm{E}$ \\
\hline \multirow[t]{7}{*}{ Cynoglossidae } & Symphurus plagiusa $\mathbf{\square}$ & $\mathrm{Sp}$ & 0.1 & 0.0 & 11.1 & 11.2 & EM \\
\hline & Número total de especies & & 22 & & & & \\
\hline & Especies dominantes & & 6 & & & & \\
\hline & Contribución al número (\%) & & 77.8 & & & & \\
\hline & Contribución al peso $(\%)$ & & 87.9 & & & & \\
\hline & Especies exclusivas en LC $\bullet$ & & & & & & \\
\hline & Especies exclusivas en LG a & & & & & & \\
\hline
\end{tabular}

*Clasificación ecológica de las especies de acuerdo con Castro-Aguirre et al. (1999).

Índice de Valor de Importancia (IVI) ( $\mathrm{N}=$ abundancia, $\mathrm{W}=$ peso, $\mathrm{F}=$ frecuencia). Se indican las abreviaturas (AB) utilizadas en la figura 7 (Análisis de Correspondencia Canónica) y la clasificación ecológica de las especies (CE*) (EM=eurihalino marino, $\mathrm{ESM}=$ estenohalino marino, $\mathrm{E}=$ estuarino, $\mathrm{D}=$ dulceacuícola).

Importance Value Index (IVI) $(\mathrm{N}=$ abundance, $\mathrm{W}=$ weight, $\mathrm{F}=$ frequency). Abbreviations (AB) used in figure 7 are shown (Canonical Correspondence Analysis) and the ecological classification of the species (CE*) (EM=euryhaline, $\mathrm{ESM}=$ estenohaline, $\mathrm{E}=$ estuarine, $\mathrm{D}=$ freshwater).

La variación temporal de H', D y J'se muestra en la figura 4 . El promedio más alto de diversidad $\left(\mathrm{H}^{\prime}=1.77 \pm 0.24\right)$ se presentó en febrero (Fig. 4D) y el más bajo $(0.84 \pm 0.46)$ en septiembre (ANOVA-F=3.98, $\mathrm{p}=0.001$ ). La riqueza promedio más alta $(\mathrm{D}=2.26 \pm 0.53)$ se registró en octubre (Fig. 4E) y la menor $(\mathrm{D}=1.1 \pm 0.52)$ en septiembre (ANOVA-F $=3.54$, $\mathrm{p}=0.002)$. La equitatividad promedio más alta $\left(\mathrm{J}^{\prime}=0.82 \pm 0.11\right)$ se presentó en febrero (Fig. $4 \mathrm{~F})$, mientras que la más baja $\left(\mathrm{J}^{\prime}=0.63 \pm 0.4\right)$ en diciembre. No se presentaron diferencias significativas para J' ( $p>0.05)$. Los valores promedio de diversidad entre épocas del año se muestran en el cuadro 1. El análisis de varianza no mostró diferencias significativas entre estas $(\mathrm{p}>0.05)$.

Con base en la variación espacio-temporal de H', D y J' en el sistema, el análisis cluster indicó la afinidad de tres grupos (Fig. 3B). El primero integrado por las estaciones 1 y 2 presentes en LC, se caracterizó por presentar los valores promedio más bajos de los índices. El segundo grupo, incluyó las estaciones 3, 4, 6 y 8 tanto para LC como para LG, con valores intermedios de H', D y J'. El tercer grupo se formó por las estaciones 5 y 7 ubicadas en LG, con valores más altos de los parámetros de la comunidad. El análisis de varianza indicó diferencias significativas entre estos grupos para los tres índices comparados (Cuadro 4).

Variación espacial y temporal de la abundancia: La densidad (ind. $/ \mathrm{m}^{2}$ ), biomasa $\left(\mathrm{g} / \mathrm{m}^{2}\right)$ y peso promedio ( $\mathrm{g} / \mathrm{ind}$.) se presenta en la figura 5, dichos parámetros no reflejaron cambios marcados en el gradiente ambiental espacial o entre grupos de estaciones para LC y LG, por lo que no se presentaron diferencias significativas $(p>0.05)$ (Cuadro 2). De acuerdo con esto, el valor promedio más alto en densidad (0.024ind. $\left./ \mathrm{m}^{2} \pm 0.02\right)$ se registró en la estación 2 y el más bajo $\left(0.012\right.$ ind. $/ \mathrm{m}^{2} \pm 0.01$ ) en las estaciones 4 y 7 (Fig. 5A). Las especies 

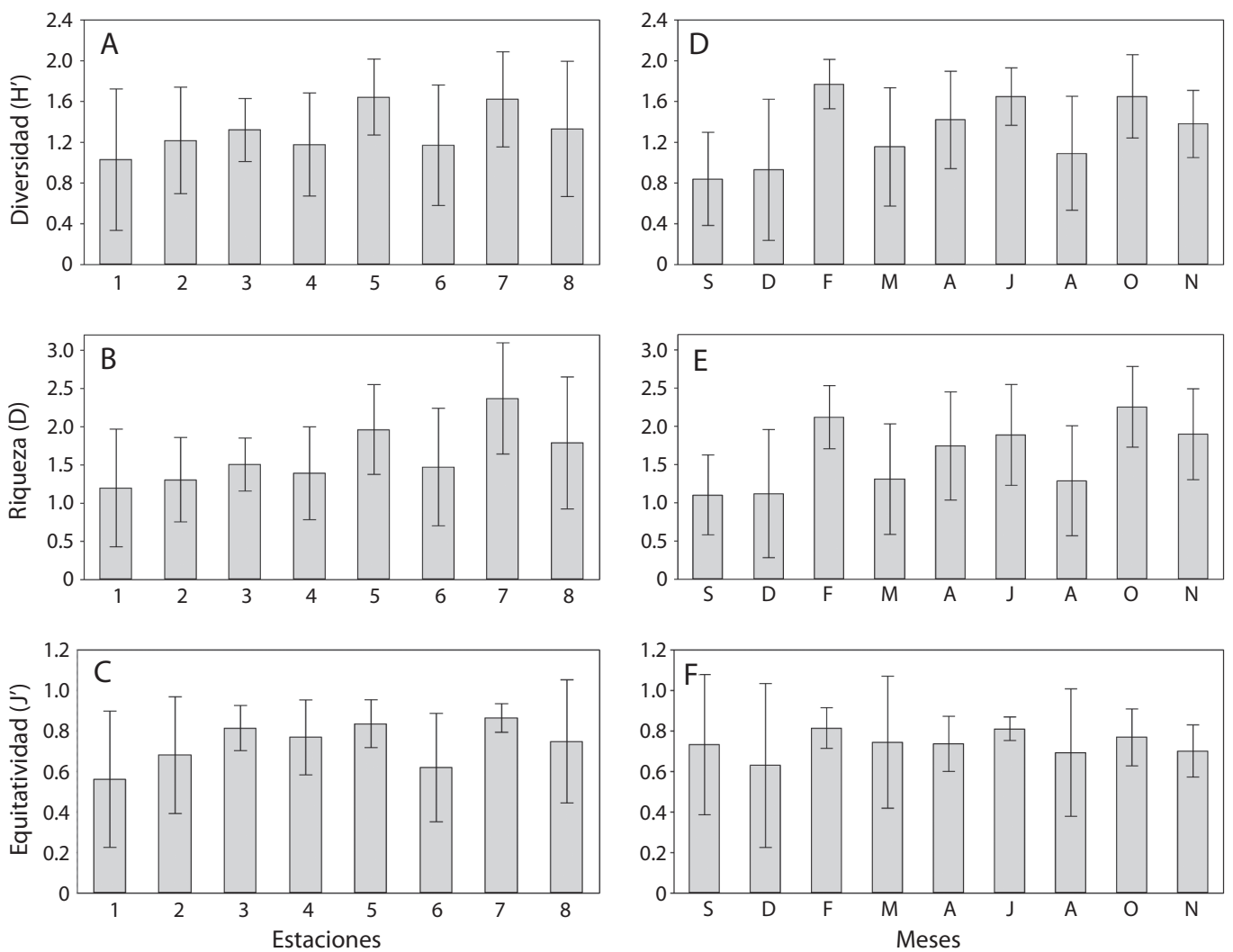

Fig. 4. Variación espacial y temporal de los índices de diversidad H', D y J' de la comunidad de peces en el sistema ChicaGrande.

Fig. 4. Spatial and temporal variation in the diversity indices H', D and J' for the fish community in the Chica-Grande system.

\section{CUADRO 4}

Valores promedio y estimadores estadísticos de los índices de diversidad calculados para cada uno de los grupos cluster de estaciones en el sistema lagunar Chica-Grande, Veracruz

TABLE 4

Average values and statistical estimators of diversity indices calculated for each one of the cluster stations groups in the Chica-Grande system lagoon, Veracruz

\begin{tabular}{cccccc} 
Parámetro & Primer grupo & Segundo grupo & Tercer grupo & Estimador & $\mathrm{p}$ \\
H' & 1.12 & 1.25 & 1.63 & $\mathrm{~F}=4.89$ & 0.010 \\
$\mathrm{D}$ & 1.25 & 1.54 & 2.17 & $\mathrm{~F}=9.08$ & $<0.0001$ \\
$\mathrm{~J}$ & 0.62 & 0.74 & 0.85 & $\mathrm{H}=8.13$ & 0.017 \\
\hline
\end{tabular}

que presentaron mayores densidades fueron: C. parallelus, D. auratus y D. rhombeus. La biomasa promedio varió de $1.011 \mathrm{~g} / \mathrm{m}^{2}$ en la estación $3 \pm 2.07$ a $0.303 \mathrm{~g} / \mathrm{m}^{2} \pm 0.2$ en la estación 7 (Fig. 5B). Las especies $O$. niloticus, $C$. parallelus y E. plumieri registraron la mayor biomasa, mientras que D. maculatus, E. melanopterus y $S$. plagiusa los valores más bajos. El peso promedio de los peces presentó su valor más alto en la estación $8(32.4 \mathrm{~g} / \mathrm{ind} . \pm 24.5)$ y 

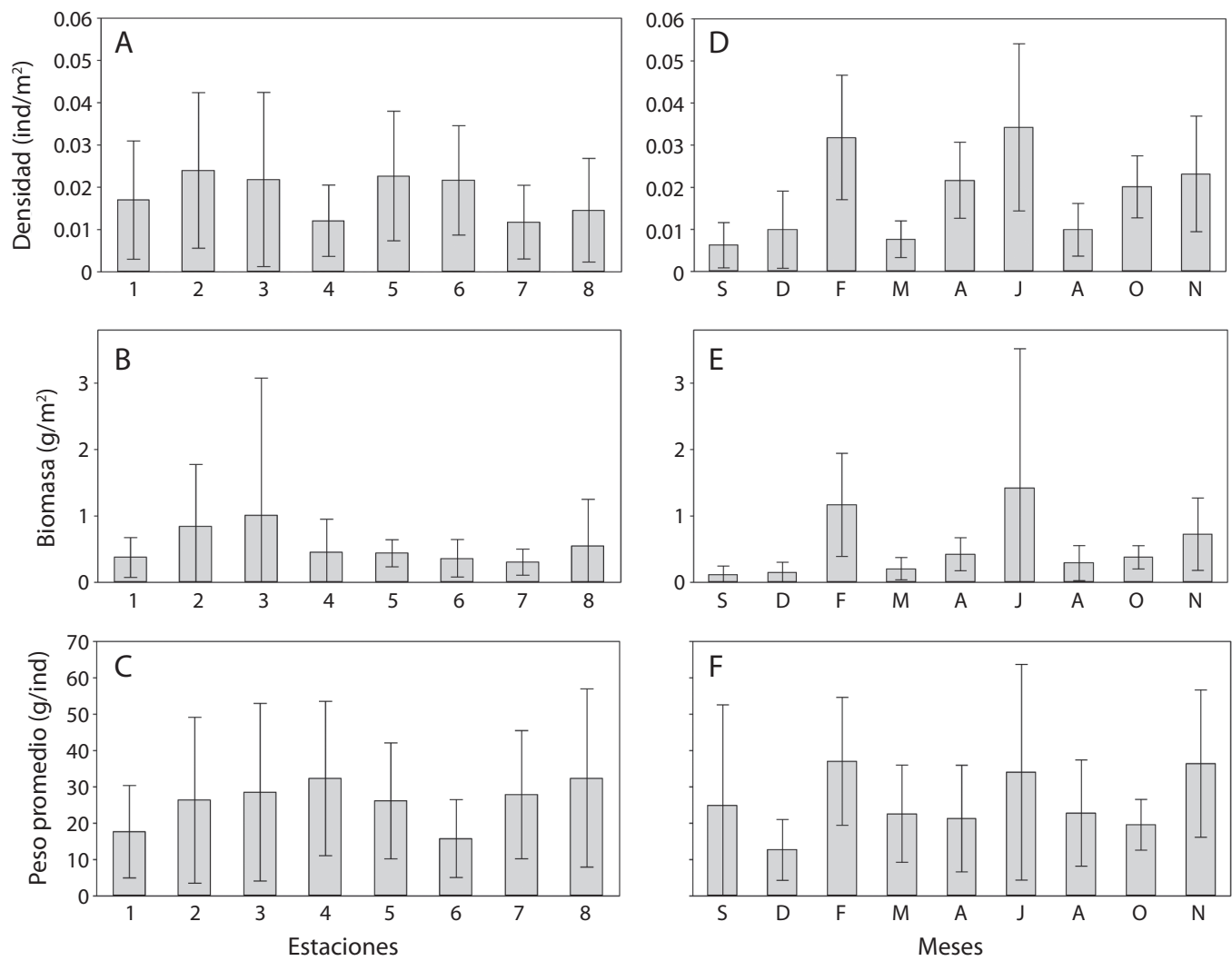

Fig. 5. Variación espacial y temporal de la densidad, biomasa y peso promedio de la comunidad de peces en el sistema Chica-Grande.

Fig. 5. Spatial and temporal variation in density, biomass and mean weight for the fish community in the Chica-Grande system.

el más bajo en la estación $6(15.8 \mathrm{~g} /$ ind. \pm 10.7$)$ (Fig. 5C).

En escala temporal los valores promedio más altos en densidad (0.034ind. $/ \mathrm{m}^{2} \pm 0.02$ ) y biomasa $\left(1.420 \mathrm{~g} / \mathrm{m}^{2} \pm 2.1\right)$ se registraron en junio y los más bajos $\left(0.005\right.$ ind. $/ \mathrm{m}^{2} \pm 0.006 \mathrm{y}$ $0.116 \mathrm{~g} / \mathrm{m}^{2} \pm 0.13$ ) en septiembre (Fig. 5D y $5 \mathrm{E}$ ). El ANOVA indicó diferencias significativas para la densidad $(\mathrm{F}=5.58, \mathrm{p}<0.0001)$ y la biomasa $(\mathrm{F}=6.77, \mathrm{p}<0.0001)$. El peso promedio presentó el valor más alto $(37.0 \mathrm{~g} / \mathrm{ind} . \pm 17.6)$ en febrero y el más bajo (12.7 g/ind. \pm 8.4$)$ en diciembre (Fig. 5F). En junio y noviembre sus valores fueron parecidos a febrero, no obstante, no se encontraron diferencias significativas entre meses $(p>0.05)$. Relacionado con este parámetro, durante abril-junio se registraron las tallas más grandes de especies como $A$. lineatus (120-150mm LT), A. probatocephalus (107-214 mm LT), C. parallelus (122270mm LT), C. spilopterus (171-198mm LT), D. rhombeus (114-170mm LT), O. niloticus (195-365mm LT) y T. maculatus $(170-180 \mathrm{~mm}$ LT) que corresponden a organismos preadultos y adultos, mientras que las tallas jóvenes $(<150 \mathrm{~mm} \mathrm{LT})$ se registraron principalmente en noviembre-febrero. Los valores promedio de abundancia entre épocas del año se muestran en el cuadro 1. El análisis de varianza no mostró diferencias significativas entre estas $(p>0.05)$.

Especies dominantes: El índice del valor de importancia (IVI) indicó que seis especies se pueden considerar como dominantes para el 
sistema Chica-Grande, las cuales representan el $77.8 \%$ de la abundancia numérica, el $87.9 \%$ de la captura total en peso y alta frecuencia de aparición en la comunidad (Cuadro 3). Las especies estuarinas que presentaron valores altos del IVI fueron Cathorops melanopus, $C$. parallelus, D. auratus, D. rhombeus y E. plumieri. Asimismo, el IVI indicó a $O$. niloticus como dominante, la cual es una especie exótica introducida en décadas pasadas y común en las áreas de influencia dulceacuícola del sistema.

Relaciones especies-hábitat: El análisis de correspondencia canónica (ACC) mostró que la relación entre las especies de peces y las variables ambientales fue explicada en un $68 \%$ de la varianza total con autovalores de 0.232 (eje 1) y 0.120 (eje 2). El diagrama de ordenación (Fig. 6) muestra la relación entre especies, los sitios y las variables ambientales; así como la importancia relativa de cada variable ambiental que determinan la composición y distribución de los peces en la comunidad. El ACC explicó el $45 \%$ de la variación especiesambiente para el eje 1, siendo la salinidad $(\mathrm{r}=0.96)$, transparencia $(\mathrm{r}=0.83)$ y temperatura $(\mathrm{r}=0.81)$ los factores ambientales que determinan la distribución de las especies a lo largo de este eje. Las especies que mostraron alta correlación con el eje 1, presentaron abundancias intermedias y bajas como C. melanopus, Citharichthys spilopterus, D. rhombeus, Gobionellus oceanicus, Hemicaranx amblyrhyncus, Mugil curema y $O$. beta, asociadas a zonas someras de mayor salinidad y transparencia (est. 5, 6, $7,8)$ con alto contenido de oxígeno disuelto. El eje 2 explicó el $23 \%$ de la variación especiesambiente, donde la profundidad $(\mathrm{r}=0.69)$ fue el factor más importante en la distribución de las especies a lo largo de este eje. Las especies que mostraron alta correlación con el eje 2 corresponden al componente dulceacuícola como $D$. maculatus, $O$. niloticus y $P$. fenestratus y al estuarino como Achirus lineatus, C. parallelus,

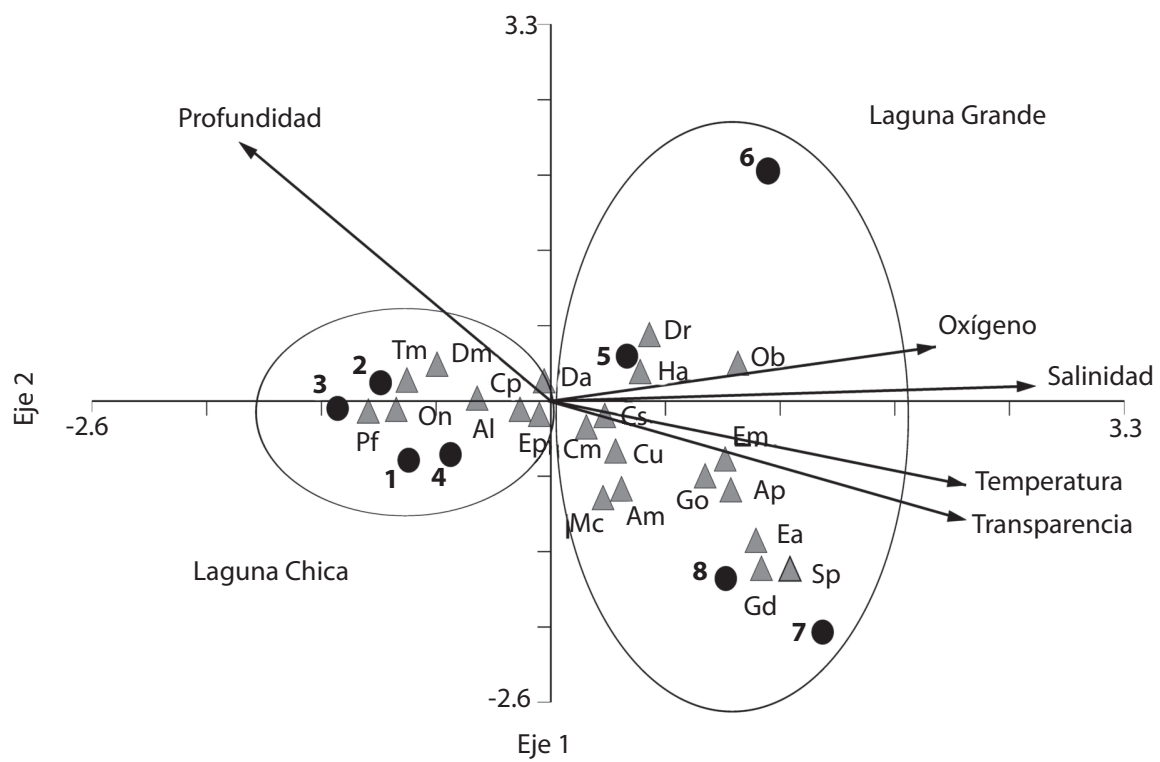

Vector scaling: 2.80

Fig. 6. Diagrama del Análisis de Correspondencia Canónica de la abundancia de las especies, las variables ambientales y los sitios de muestreo en el sistema lagunar Chica-Grande. La longitud y dirección de las flechas indican la importancia relativa de las variables ambientales. Las abreviaturas de las especies (AB) se indican en el cuadro 3.

Fig. 6. Canonical Correspondence Analysis Plot of species abundance, environmental variables and sampling sites in the Chica-Grande lagoon system. Arrow length and direction indicate the relative importance of environmental variables. Species abbreviations $(\mathrm{AB})$ are listed in table 3 . 
D. auratus, E. plumieri y Trinectes maculatus. Estas especies presentaron abundancias intermedias y altas, asociadas a zonas profundas con valores bajos de salinidad, transparencia y oxígeno disuelto (est. 1, 2, 3, 4).

\section{DISCUSIÓN}

Diversos estudios en sistemas lagunares costeros han señalado que la variación ambiental en escala espacial y temporal es muy marcada debido a diversos factores, como el balance determinado por la descarga de agua dulce de los ríos y la entrada de agua marina, los cambios climáticos, la superficie y geomorfología del sistema, la presencia de canales sinuosos, el tamaño de la boca de conexión con el mar, la amplitud de las mareas, las corrientes costeras e interiores, el grado de insolación, entre otros (Lara-Domínguez et al., 2011a; Snedden et al., 2012). Esta variación ambiental influye en la estructura y organización de las comunidades de peces en estos sistemas, modificando la composición, riqueza, distribución y abundancia de las especies en espacio y tiempo (Marshall \& Elliot, 1998; Araújo et al., 2002; Barletta et al., 2005; Pombo et al., 2005; Azevedo et al., 2007; Aguirre-León, Díaz-Ruiz, RamírezHuerta, 2010; Arceo-Carranza et al., 2010).

En el sistema lagunar Chica-Grande, la variabilidad de los parámetros físico-químicos está relacionada principalmente con la mayor descarga de los ríos en la porción norte (Laguna Chica) y la entrada de agua marina en el sur (Laguna Grande) determinadas por los cambios estacionales en las distintas épocas climáticas de la región (CNA, 2003; PérezMaqueo, Muñoz-Villers, Vázquez, Equihua \& León-Romero, 2011). El sistema presentó un gradiente salino que varió desde condiciones marinas con alta concentración de oxígeno y transparencia en el extremo sur, a condiciones dulceacuícolas con los menores valores de estos parámetros en la región norte del sistema. Las diferencias estadísticas significativas de los parámetros físico-químicos y el análisis de similitud entre sitios permitieron caracterizar al sistema con dos hábitats diferentes, uno de menor salinidad y oxígeno disuelto en la porción norte, y otro de mayor salinidad y oxígeno disuelto en la porción sur del sistema. Esta condición se ve favorecida por la presencia de un canal estrecho y sinuoso que conecta al sistema con el mar, y a la geomorfología que divide al mismo en dos pequeños cuerpos de agua (Lagunas Chica y Grande) separados por un estrecho canal (El Caño). Durante marzo y junio (secas) la salinidad fue máxima como resultado del incremento en la insolación y la evaporación, así como la disminución de drenaje de los ríos en el sistema, mientras que la mínima se presentó en diciembre y febrero (nortes) debido a la influencia de los vientos de invierno que mueven el agua dulce proveniente de los afluentes hacia la boca de conexión y atenúan el efecto de la marea (Aguirre-León et al., 2010). Con base en la variación espaciotemporal de la salinidad, el sistema es en general mesohalino (5-18ups) con cambios a oligohalino (3.4-5.0ups) en la porción norte durante diciembre, a polihalino (20.3-20.8ups) en el sur durante junio. Mecalco-Hernández (2010) registró resultados similares en este sistema, señalando condiciones mesohalinas para algunos meses del año con valores de 10-18ups.

Los cambios de la temperatura del agua indicaron poca variación debido a la incidencia solar y a las condiciones de mezcla vertical como reflejo de la poca profundidad del sistema (Snedden et al., 2013), por lo cual, la diferencia entre sitios fue pequeña $\left(3.2^{\circ} \mathrm{C}\right)$ y no significativa. La variación de la temperatura se relacionó con las temporadas climáticas del año, donde los meses de primavera-verano son los más cálidos y otoño-invierno los más fríos. Este patrón coincide en otros ecosistemas similares del Golfo de México (Lara-Domínguez et al., 2011a).

El oxígeno disuelto puede actuar como un parámetro que favorece o limita la presencia y/o la ausencia de organismos en los sistemas acuáticos costeros (Marshall \& Elliott, 1998; Azevedo et al., 2007). Las variaciones espaciales de este parámetro en Chica-Grande pueden deberse a los procesos de corriente y mezcla que ocurren en mayor grado en la parte sur por 
su cercanía a la boca de conexión con el mar y a la actividad de organismos fotosintéticos y otros productores asociados (algas rodófitas en LG). El oxígeno disuelto presentó también un patrón relacionado con la estacionalidad climática, con mayor concentración durante los nortes, y la menor en secas e inicio de lluvias. Esta tendencia se relaciona a su vez con el cambio temporal de la temperatura del agua y al aporte de materia orgánica por los ríos (de la Lanza, Rodríguez, Baus \& Pulido, 2011).

La profundidad en lagunas costeras está asociada al régimen de precipitación pluvial, a la geomorfología del sistema, a la distancia de influencia marina y a la apertura y cierre de la boca de conexión con el mar (Lara-Domínguez et al., 2011a). Al respecto, el nivel del agua más bajo en los sitios de estudio se presentó al final de secas e inicio de la época de lluvias. Mientras que, los niveles máximos de agua registrados en el sistema se presentaron durante las lluvias (septiembre y octubre) debido al máximo incremento del caudal por la precipitación en la zona norte del sistema provocado por el paso del huracán "Rita" en esa temporada.

El sistema lagunar Chica-Grande presentó características hidrológicas similares a otras lagunas de la costa de Veracruz, con gradientes ambientales espaciales y temporales marcados y geomorfología parecida, cuya dinámica ambiental depende de la descarga de los ríos, la amplitud de marea, la longitud y forma de los canales de comunicación con el mar y del tamaño de la boca con el mar (Díaz-Ruiz, Pérez-Hernández \& Aguirre-León, 2003; Castillo-Rivera, Zárate-Hernández \& Ortíz-Burgos, 2005; Lara-Domínguez et al., 2011b). Estos estudios han indicado que las variables ambientales tales como la salinidad, temperatura, profundidad, entre otras, influyen en las diferentes especies de peces, ya que las lagunas costeras son áreas que utilizan durante diversas etapas de su ciclo de vida y con diferentes requerimientos biológicos. Esto permite explicar los cambios en la diversidad de los ensambles de peces en los distintos sectores de estos ecosistemas, en respuesta a la dinámica espacio-temporal de la abundancia, biomasa, distribución y frecuencia de aparición de las distintas especies de peces (Díaz-Ruiz et al., 2012).

En este trabajo el número de especies registradas fue menor a las reportadas por Bedia-Sánchez \& Franco (2008) con 33 especies y Arzola-Vargas \& Bautista-Chávez (2010) con 40 especies para el sistema lagunar ChicaGrande. Con base en las especies identificadas en el presente estudio y una síntesis de la literatura que incluye registros específicos del sistema Chica-Grande, se estima que esta laguna costera contiene al menos 72 especies (Kobelkowsky, 1991; Reséndez-Medina \& Kobelkowsky, 1991; Lozano-Vilano, García-Ramírez \& Contreras-Balderas, 1993; Castro-Aguirre et al., 1999; Bedia-Sánchez \& Franco, 2008; Arzola-Vargas \& BautistaChávez, 2010). Sin embargo, es razonable suponer que el número de especies que utilizan dicho cuerpo lagunar pueda ser mayor. No obstante, el número total de especies identificadas en el presente estudio, se incluyen nuevos registros o actualizaciones sinonímicas como: P. fenestratus, E. amblyopsis, G. oceanicus y $S$. plagiusa. Miller (2009) reportó a P. fenestratus como especie válida con distribución geográfica costera en Veracruz cercana al sistema Chica-Grande. McMahan, Geheber \& Piller (2010) la incluyen como resultado de un nuevo acomodo genérico basado en la determinación de su citocromo mitocondrial. Froese \& Pauly (2012) y Eschmeyer (2013) también incluyen a $P$. fenestratus en sus registros de peces. Respecto a E. amblyopsis, Castro-Aguirre et al. (1999) la reportó por primera vez para las lagunas de Tamihua y Tampamachoco, al norte de Chica-Grande en Veracruz. G. oceanicus fue reportado en trabajos anteriores con el sinónimo de G. hastatus (Froese \& Pauly, 2012, Eschmeyer, 2013). Debe señalarse también que pocas especies estuvieron presentes únicamente en alguna de las lagunas debido a las características ambientales del hábitat, como es el caso de $P$. fenestratus registrada sólo en Laguna Chica con baja salinidad y $O$. beta capturada sólo en Laguna Grande con salinidad más alta $\mathrm{y}$ asociada a fondos con vegetación sumergida. 
En el sistema lagunar Chica-Grande, la diversidad y abundancia de los peces estuvo relacionada con la salinidad, temperatura y transparencia. Espacialmente, la diversidad y riqueza de peces fueron significativamente superiores en sitios con salinidad y temperaturas mayores y con vegetación sumergida. Dichos sitios corresponden a la parte sur del sistema (Laguna Grande) con características estuarinas y vegetación sumergida, las cuales influyen en la presencia de especies estacionales y ocasionales estenohalinas, debido a que representan áreas de alta disponibilidad de alimento, crecimiento y protección de depredadores. Diversos estudios realizados en lagunas costeras y estuarios subtropicales y tropicales, han señalado que las variables ambientales (i.e. salinidad, temperatura y transparencia, entre otras) están relacionadas con la diversidad de los ensambles de peces (Barletta et al., 2005; Azevedo et al., 2007; Arceo-Carranza et al., 2010; Castillo-Rivera, Ortíz-Burgos \& ZárateHernández, 2011; Díaz-Ruiz et al., 2012).

En el sistema Chica-Grande, la diversidad y riqueza de especies mostraron una relación estacional con la salinidad y la temperatura con valores altos en octubre-febrero y bajos en septiembre. Estudios realizados en la región costera del Golfo de México indican alta diversidad de peces durante la época de lluvias por el aporte de nutrientes de los ríos e incremento de la producción primaria (Díaz-Ruiz et al., 2003; Aguirre-León et al., 2010; Arceo-Carranza et al., 2010; Castillo-Rivera et al., 2011). En este sistema, el patrón de baja diversidad que se presentó en septiembre (lluvias), puede relacionarse con la época de huracanes que ocurrió en la región. Mientras que, la mayor diversidad ( $\mathrm{H}^{\prime}$, D) en el mes de noviembre (nortes) se debió a la presencia de especies dulceacuícolas y eurihalinas marinas que incursionaron dentro del estuario en esta época del año, cuando la salinidad disminuye por efecto de empuje de agua dulce hacia la boca de conexión por acción del viento (Aguirre-León et al., 2010).

Los patrones de abundancia de las poblaciones están influenciados por los cambios en las condiciones ambientales del sistema, así como por los patrones de migración estacional de las especies durante sus ciclos de vida (Miller \& Skilleter, 2006; Azevedo et al., 2007; Aguirre-León et al., 2010; Arceo-Carranza et al., 2010; Lara-Domínguez et al., 2011b; DíazRuiz et al., 2012). La biomasa y densidad de los peces incrementó durante junio cuando la salinidad, temperatura y transparencia fueron altas, lo cual sugiere que las especies marinas y estuarinas se desplazan en la época de secas del mar a la laguna como preadultos y adultos $(>150 \mathrm{~mm}$ LT) para alimentarse y/o protegerse, como A. lineatus, A. probatocephalus, $C$. parallelus, C. spilopterus, D. rhombeus y $T$. maculatus. Por otra parte, individuos jóvenes $(<150 \mathrm{~mm}$ LT) la utilizan durante agosto-octubre (lluvias) donde se alimentan y crecen, lo cual se corroboró por la frecuencia de tallas y la disminución de los valores espacio-temporales de la densidad, biomasa y peso promedio de los peces. Especies del componente dulceacuícola y estuarino como $P$. fenestratus, $O$. niloticus y E. amblyopsis son abundantes durante las lluvias y pasan todo o parte de su ciclo de vida en el sistema, principalmente en la zona norte de la laguna. La abundancia en el sistema ChicaGrande, sugiere que los cambios ontogenéticos de las especies están relacionados con la variación ambiental del sistema, lo que favorece la utilización espacial y temporal del ecosistema a través de sus patrones de migración, ocasionando cambios en la estructura de la comunidad de peces en este sistema (Miller \& Skilleter, 2006; Azevedo et al., 2007; Aguirre-León et al., 2010; Arceo-Carranza et al., 2010; Lara-Domínguez et al., 2011b; Díaz-Ruiz et al., 2012).

En el sistema Chica-Grande C. parallelus, E. plumieri y $O$. niloticus son un recurso pesquero local en esta región (Lara-Domínguez et al., 2011b). Por su alta abundancia, frecuencia y distribución es evidente el éxito ecológico de las especies dominantes en este sistema, lo cual indica que han adaptado sus estrategias alimenticias, reproductivas, de crianza o protección, lo que favorece su permanencia en el ecosistema (Aguirre-León et al., 2010; Lara-Domínguez et al., 2011b; Díaz-Ruiz et al., 2012). Las fluctuaciones temporales de los parámetros 
ambientales en los diferentes hábitats, tienen su principal influencia en la distribución y abundancia de las especies dominantes. Tal es el caso de $C$. parallelus que se alimenta como preadulto y adulto durante junio y noviembre, en áreas de manglar de la zona norte del sistema cuando la salinidad y la temperatura son altas. Mientras que tallas de individuos jóvenes se registraron en lluvias (agosto-octubre) asociados al hábitat de macroalgas en la zona sur del sistema. Castillo-Rivera, Zavala-Hurtado, Zárate-Hernández (2002) observaron una dinámica similar para esta especie en el sistema Pueblo Viejo al norte de Veracruz, Golfo de México. D. auratus, D. rhombeus y E. plumieri, son especies con dependencia estuarina y gran capacidad euritermohalina, las cuales realizan migraciones entre los ambientes del sistema con fines de alimentación (AguirreLeón et al., 2010). En Chica-Grande se distribuyen preferentemente en ambientes de mayor salinidad del sistema, donde utilizan las áreas de vegetación sumergida, predominando los jóvenes durante los nortes y secas. Las especies de la familia Gerreidae, también muestran este patrón en otras lagunas costeras estudiadas en el Golfo de México por Aguirre-León \& YáñezArancibia (1986), Aguirre-León \& Díaz-Ruiz (2000, 2006), Pérez-Velázquez, Aguirre-León \& Díaz-Ruiz (2007), Aguirre-León et al. (2010), Arceo-Carranza et al. (2010), Castillo et al. (2011). Al igual que otras especies dominantes, C. melanopus se distribuye preferentemente en áreas someras de menor salinidad y transparencia como adulto en agosto-octubre y realiza migraciones cortas de carácter trófico y crianza que optimiza la variación temporal del ambiente. El éxito de adaptación de $C$. melanopus en ambientes de baja salinidad, profundidad y alta turbidez ha sido informado también por Lara-Domínguez, Yáñez-Arancibia \& Amezcua-Linares (1981), Yáñez-Arancibia \& Lara-Domínguez (1988), Aguirre-León et al. (2010). Los resultados mostraron a $O$. niloticus del componente dulceacuícola como dominante en el sistema, la cual presentó alta frecuencia de aparición, sin embargo, la mayor abundancia se registró en ambientes de baja salinidad y mayor descarga de los ríos. Esta especie exótica o invasora fue introducida en México en 1964 (Froese \& Pauly, 2012) y posteriormente en Veracruz (Arredondo-Figueroa \& Lozano, 1996). La liberación intencionada y su dispersión al medio natural han provocado controversias a nivel mundial, acerca de si esta especie ocasiona algún impacto en las especies nativas de sistemas costeros (Iongh \& VanZon, 1993; McKaye, Ryan, Stauffer, López-Pérez \& Berghe, 1995). En Chica-Grande no existe ningún estudio que aborde esta problemática y hasta el momento se desconoce el efecto que está teniendo, no obstante, la pesquería de las tilapias se considera importante, ya que representa un recurso económico local. Esta especie también ha sido reportada en las listas ictiofaunísticas por Castillo-Rivera et al. (2005), Chávez-López et al. (2005), Díaz-Ruiz et al. (2003), Velázquez-Velázquez, Vega-Cendejas \& Navarro-Alberto (2008) entre otros, sin que se hayan evaluado las consecuencias de su introducción en lagunas costeras del país.

El análisis de correspondencia canónica realizado para el sistema Chica-Grande, reforzó que las lagunas Chica y Grande se diferencian por sus características ambientales particulares. Al mismo tiempo que la comunidad de peces responde a dichas características en cada hábitat dentro del sistema a través del tiempo, lo cual se refleja en una estructura particular de conjuntos de poblaciones y especies dominantes. Es razonable suponer, que las variaciones en la composición de peces, se deben a la diferente capacidad eurihalina y euritérmica de las especies que les permiten utilizar diferentes hábitats específicos. Esto es común en estuarios y lagunas costeras de zonas tropicales, donde las especies han adaptado estrategias de uso del sistema a través de sus ciclos de vida (Azevedo et al., 2007; Aguirre León et al., 2010; Díaz-Ruiz et al., 2012). En este sistema los ensambles de peces estuvieron estrechamente relacionados a los cambios de salinidad durante la época de secas (marzo-junio) y lluvias (agosto-octubre). Se observó un grupo caracterizado por especies marinas y estuarinas que están relacionadas a las áreas someras de 
mayor salinidad, transparencia y oxígeno $(C$. spilopterus, D. rhombeus, G. oceanicus, $H$. amblyrhyncus, M. curema y O. beta). Estas especies utilizan las zonas de mayor influencia marina, vegetación sumergida y manglar de la región sur del sistema durante marzo-junio. Mientras que otras especies del componente dulceacuícola (D. maculatus, O. niloticus y $P$. fenestratus) y estuarino (A. lineatus, C. melanopus, C. parallelus, D. auratus, E. plumieri y $T$. maculatus) están estrechamente relacionadas con los cambios de salinidad durante las lluvias y nortes. Estas especies coexisten en áreas de mayor influencia fluvial y menor transparencia en zonas más profundas donde realizan parte de sus ciclos de vida. Los resultados sugieren que las variaciones estacionales de los factores abióticos en las temporadas de secas y lluvias se relacionan con la descarga de los ríos, lo cual se refleja en la estructura de los conjuntos de peces. Los ensambles de peces observados en este estudio, son similares a los encontrados en otros sistemas costeros del Golfo de México (Díaz-Ruiz et al., 2003; Aguirre-León et al., 2010; Arceo-Carranza et al., 2010; Castillo et al., 2011; Lara-Domínguez et al., 2011b), esto indica que las asociaciones de especies están correlacionadas con la dinámica ambiental de los hábitats que prevalecen en los sistemas, a través del establecimiento de patrones de migración y/o colonización de los hábitats costeros mediante interacciones entre la plataforma continental y los ecosistemas de aguas protegidas.

\section{AGRADECIMIENTOS}

Al Departamento de Hidrobiología de la Universidad Autónoma Metropolitana Iztapalapa (Proyecto No. CBS-147.05.05) el apoyo institucional y económico para realizar este estudio. Los autores agradecen a todos los estudiantes su colaboración en las campañas de campo y laboratorio. Se agradece a los árbitros sus comentarios y sugerencias realizadas.

\section{RESUMEN}

Las lagunas costeras de Veracruz en el Golfo de México, incluyen gran variedad de recursos biológicos, por lo que requieren mayor conocimiento ecológico, especialmente para sus comunidades de peces. Esta investigación analizó la variación espacial y temporal de la diversidad, abundancia y asociaciones de la comunidad de peces y su relación con los parámetros físico-químicos del sistema lagunar Chica-Grande en Veracruz. Para esto, en ocho estaciones se muestrearon las características del agua y la composición de la comunidad de peces (lances de 10min con red de arrastre camaronera) entre septiembre 2005 y noviembre 2006. La variación espacio-temporal de los parámetros físico-químicos, permitió definir dos ambientes contrastantes de acuerdo con los gradientes de salinidad, temperatura, transparencia, oxígeno disuelto y profundidad. Se recolectaron un total de 1947 peces con un peso de $57.88 \mathrm{~kg}$. De estos, se identificaron 22 especies, 20 géneros y 14 familias; y cuatro especies son nuevos registros para el sistema. Como se observó para las condiciones físicoquímicas, la diversidad de la comunidad de peces también mostró un gradiente espacial, con valores altos $\left(\mathrm{H}^{\prime}=2.37\right.$, $\left.\mathrm{D}=3.35, \mathrm{~J}^{\prime}=0.82\right)$ en el hábitat salobre y bajos en el ambiente más dulce. La abundancia de los peces no reflejó cambios marcados en el gradiente, no obstante, fue mayor en el hábitat más dulce. Durante los meses de "nortes" (Noviembre-Febrero) la diversidad $\left(\mathrm{H}^{\prime}=2.05\right)$ y riqueza de especies $(\mathrm{D}=2.99)$ fue más alta, mientras que la densidad y biomasa fue mayor $\left(0.034\right.$ ind. $\left./ \mathrm{m}^{2}, 1.42 \mathrm{~g} / \mathrm{m}^{2}\right)$ durante los meses de lluvias (Julio-Octubre). El Índice de Valor de Importancia (IVI) definió 6 especies dominantes que representan el $77.8 \%$ de la abundancia numérica y el $87.9 \%$ de la captura total en peso. El Análisis de Correspondencia Canónica (ACC) mostró que la relación peces-hábitat fue explicada en un $68 \%$ de la varianza total para los primeros dos ejes, donde los cambios de salinidad, transparencia y temperatura fueron los factores ambientales que determinaron la composición y distribución de los peces en la comunidad. En este estudio, los gradientes espaciales de las variables ambientales permitieron explicar los cambios en la composición y abundancia de las especies, mientras que los temporales, la estructura comunitaria y su relación con los patrones estacionales de utilización de hábitats por las especies durante sus ciclos de vida.

Palabras clave: heterogeneidad ambiental, estructura comunitaria, relaciones peces-hábitat, Laguna Chica-Grande, Veracruz, México.

\section{REFERENCIAS}

Able, K. W. (2005). A re-examination of fish estuarine dependence: Evidence for connectivity between 
estuarine and ocean habitats. Estuarine, Coastal and Shelf Sciences, 64, 5-17

Aguirre-León, A., \& Yáñez-Arancibia, A. (1986). Las mojarras de la Laguna de Términos: Taxonomía, biología, ecología y dinámica trófica (Pisces: Gerreidae). Anales del Instituto de Ciencias del Mar Limnología, 13, 369-444.

Aguirre-León, A., \& Díaz-Ruiz, S. (2000). Population structure, gonadal maturity and feeding habits of Eugerres plumieri (Gerreidae) in the Pom-Atasta fluvial-deltaic system, Mexico. Ciencias Marinas, 26, 253-273.

Aguirre-León, A., \& Díaz-Ruiz, S. (2006). Estructura de tallas, madurez gonádica y alimentación de Diapterus rhombeus (Gerreidae) en el sistema fluvio-deltaico Pom-Atasta, Campeche, México. Revista de Biología Tropical, 54, 599-611.

Aguirre-León, A., Díaz-Ruiz, S., \& Ramírez-Huerta, A. (2010). Ecología de peces dominantes costeros tropicales. Estudio para el sistema fluvio-deltaico Pom-Atasta, Campeche. Serie Académicos No. 96. México: CBS, UAM-Xochimilco.

Araújo, F. G., Azevedo, M. C. C., Silva, M. A., Pessanha, Gomes, A. L. M. I. D., \& Cruz-Filho, A. G. (2002). Environmental influences on the demersal fish assemblages in the Sepetiba Bay, Brazil. Estuaries, $25,441-450$.

Arceo-Carranza, D., Vega-Cendejas, M. E., MonteroMuñoz, J. L., \& Hernández de Santillana, M. J. (2010). Influencia del hábitat en las asociaciones nictimerales de peces en una laguna costera tropical. Revista Mexicana de Biodiversidad, 81, 823-837.

Arredondo-Figueroa, J. L., \& Lozano, G. S. (1996). El Cultivo de la Tilapia en México. Memorias del primer curso internacional de producción de tilapia. México: UNAM, SEMARNAP.

Arzola-Vargas, X. T., \& Bautista-Chávez., A. G. (2010). Diversidad de la comunidad de peces en el sistema lagunar Laguna Grande-Laguna Chica, Vega de Alatorre, Veracruz. (Tesis de Licenciatura no publicada). Universidad Nacional Autónoma de México, Distrito Federal, México.

Azevedo, M. C. C., Araújo, F. G., Cruz-Filho, A. G., Pessanha, A. L. M, Silva, M. A. \& Guedes, A. P. P. (2007). Demersal fishes in a tropical bay in southeastern Brazil: partitioning the spatial, temporal and environmental components of ecological variation. Estuaries and Coastal Shelf Science, 75, 468-480.

Barletta, M., Barletta-Bergan, A., Saint-Paul, U., \& Hubol, G. (2005). The role of salinity in structuring the fish assemblages in tropical estuary. Journal of Fish Biology, 66, 45-72.

Barreiros, J. P., Branco, J. O., Junior, F. F., Machado, L., Hostim-Silva, M., \& Verani, J. R. (2009). Space-time distribution of the ichthyofauna from Saco da Fazenda estuary, Itajaí, Santa Catarina, Brazil. Journal of Coastal Research, 25, 1114-1121.

Bedia-Sánchez, C., \& Franco-López, J. (2008). Peces de los Sistemas Costeros del Estado de Veracruz. México: UNAM, FES Iztacala.

Castillo-Rivera, M., Zavala-Hurtado, J. A., \& ZárateHernández, R. (2002). Exploration of spatial and temporal patterns of fish diversity and composition in a tropical estuarine system of Mexico. Reviews in Fish Biology and Fisheries, 12, 167-177.

Castillo-Rivera, M., Zárate-Hernández, R., \& Ortíz-Burgos, S. (2005). Variación nictímera y estacional de la abundancia, riqueza y especies dominantes de peces, en un ambiente salobre de sustrato blando. Revista Hidrobiología, 18, 227-238.

Castillo-Rivera, M., Ortíz-Burgos, S., \& Zárate-Hernández, R. (2011). Estructura de la comunidad de peces estuarinos en un hábitat con vegetación sumergida: variación estacional y nictémera. Revista Hidrobiología, 21, 311-321.

Castro-Aguirre, J. L., Espinosa-Pérez, H., \& SchmitterSoto, J. J. (1999). Ictiofauna Estuarino-Lagunar y Vicaria de México. México: Limusa.

Cervigón, F. (1991-1994). Los peces marinos de Venezuela. Fundación Científica Los Roques. Venezuela: Universidad de Oriente.

Chávez López, R., Rocha Ramírez, A., \& Ramírez Rojas, A. (2005). Cambios en los ensamblajes de peces del Sistema Lagunar de Alvarado, Veracruz, México. Revista Digital Universitaria, 6(8), 3-19.

CNA. (2003). Programa Hidráulico Regional 2002-2006. Comisión Nacional del Agua (CNA), Región X Golfo Centro México.

De la Cruz-Agüero, G. (1994). ANACOM sistema para el Análisis de Comunidades. Versión 3.0. México: CICIMAR-IPN.

De la Lanza Espino, G., Rodríguez, I. P., Baus, S. C., \& Pulido, S. H. (2011). Química de las aguas costeras. In G. de la Lanza Espino \& S. H. Pulido (Eds.), Ambiente, Biología, Sociedad, Manejo y Legislación de Sistemas Costeros Mexicanos (pp. 141-177). México: UMSNH, WWF, Plaza y Valdés.

Díaz-Ruiz, S., Pérez-Hernández, M., \& Aguirre-León, A. (2003). Caracterización de los conjuntos de peces en una laguna costera tropical del noroeste del Golfo de México. Ciencias Marinas, 29, 631-644.

Díaz-Ruiz, S., Aguirre-León, A., Calva-Benítez, L. G., \& Barba-Macías, E. (2012). Variación estacional de la estructura comunitaria de peces en el sistema Carretas-Pereyra, Reserva de la Biosfera La Encrucijada, Chiapas. In A. J. Sánchez, X. Chiappa-Carrara \& R. Brito-Pérez (Eds.), Recursos Acuáticos 
Costeros del Sureste (pp. 191-210). Vol 2. México: CONCIYTEY-UNAM.

Eschmeyer, W. N. (2013). Catalog of Fishes. USA: California Academic of Sciences. Recuperado de http:// www.research.calacademy.org

Fischer, W. (1995). Species Identification Sheets for Fishery Purpose. Western Central Atlantic (Fishing area 31). Vol. 1-5. Rome: FAO.

Froese, R., \& Pauly, D. (2012). FishBase. Version 10/2012. Recuperado de http://www.fishbase.org

García, E. (2004). Modificaciones al Sistema de Clasificación Climática de Köppen. México: Instituto de Geografía, UNAM.

Gelwick, F. P., Akin, S., Arrington, D. A., \& Winemiller, K. O. (2001). Fish assemblage structure in relation to environmental variation in a Texas Gulf coastal wetland. Estuaries 24, 285-296.

Hair, J. F., Anderson, R. E., Tatham, R. L., \& Black, W. C. (2001). Multivariate Data Analysis. Madrid, España: Prentince-Hall.

Iongh, H. H., \& Van Zon, J. C. J. (1993). Assessment of impact of the introduction of exotic fish species in north-east Thailand. Aquaculture Fisheries Management, 24, 279-289.

Kobelkowsky, A. (1991). Ictiofauna de las lagunas costeras del estado de Veracruz. In G. Figueroa, C. Álvarez, A. Esquivel \& M. E. Ponce (Eds.), Fisicoquímica y Biología de las Lagunas Costeras Mexicanas (pp. 74-93). México: UAM-Iztapalapa.

Lara-Domínguez, A. L., Yáñez-Arancibia, A., \& AmezcuaLinares, F. (1981). Biología y ecología del bagre Arius melanopus (Günther) en la Laguna de Términos, sur del Golfo de México (Pisces: Ariidae). Anales del Instituto de Ciencias del Mar y Limnología, 81, 267-304.

Lara-Domínguez, A. L., Contreras-Espinosa, F., CastañedaLópez, O., Barba-Macías, E., \& Pérez-Hernández, M. A. (2011a). Lagunas costeras y estuarios. In A. CruzAngón (Ed.). La Biodiversidad en Veracruz: Estudio del Estado (pp. 301-317). México: CONABIO.

Lara-Domínguez, A. L., Franco-López, J., Bedia-Sánchez, C., Abarca-Arenas, L. G., Díaz-Ruiz, S., AguirreLeón, A., González-Gándara, C., \& Castillo-Rivera, M. (2011b). Diversidad de peces en los ambientes costeros y plataforma continental. In A. Cruz-Angón (Ed.), La Biodiversidad en Veracruz: Estudio del Estado (pp. 505-516). México: CONABIO.

Levene, H. (1960). Robust test for equality on variance. In I. Olkin, S. G. Ghurye, W. Hoeffding, W. G. Madow \& H. B. Mann (Eds.), Contributions to Probability and Statistics (pp. 278-292). California: Stanford University.

Lozano-Vilano, M. L., García-Ramírez, M. E., \& Contreras-Balderas, S. (1993). Peces costeros y marinos del
Estado de Veracruz. In S. S. Salazar-Vallejo \& N. E. González (Eds.), Biodiversidad Marina y Costera de México (pp. 576-595). México: CONABIO, CIQRO.

Margalef, R. (1969). Perspectives in Ecological Theory. Chicago: University of Chicago.

Marshall, S. \& Elliot, M. (1998). Environmental influences on the fish assemblages of the Humber Estuary, U.K. Estuaries and Coastal Shelf Science, 46, 175-184.

McKaye, K. R., Ryan, J. D., Stauffer, J. R., López-Pérez, L. J., \& Van Den Berghe, E. P. (1995). African tilapia in Lake Nicaragua: Ecosystem in transition. Bioscience, 45, 406-411.

McMahan, C. D., Geheber, A. D., \& Piller, K. R. (2010). Molecular systematic of the enigmatic Middle American genus Vieja (Teleostei: Cichlidae). Molecular Phylogeny and Evolution, 57, 1293-1300.

Mecalco-Hernández, A. (2010). Dinámica del bacterioplancton y su relación con la biomasa fitoplanctónica en dos lagunas costeras del estado de Veracruz. (Tesis de Maestría no publicada). Universidad Autónoma Metropolitana-Iztapalapa, Distrito Federal, México.

Miller, R. R. (2009). Peces dulceacuícolas de México. México: CONABIO.

Miller, S. J., \& Skilleter, G. A. (2006). Temporal variation in habitat use by nekton in a subtropical estuarine system. Journal of Experimental Marine Biology and Ecology, 337, 82-95.

Moreno-Casasola, P., Cejudo-Espinosa, E., CapistránBarradas, A., Infante-Mata, D., López-Rosas, H., Castillo-Campos, G., Pale-Pale, J., \& Campos-Cascaredo, A. (2010). Composición florística, diversidad y ecología de humedales herbáceos emergentes en la planicie costera central de Veracruz, México. Boletín de la Sociedad Botánica de México, 87, 29-50.

Pérez-Maqueo, O., Muñoz-Villers, L., Vázquez, G., Equihua Zamora, M. E., \& León-Romero, P. (2011). Hidrología. In A. Cruz-Angón (Ed.). La Biodiversidad en Veracruz: Estudio del Estado (pp. 289-292). México: CONABIO.

Pérez-Velázquez, P. A., Aguirre-León, A., \& Díaz-Ruiz, S. (2007). Abundancia nictemeral de Eugerres plumieri (Pisces: Gerreidae) en un sistema fluvio-deltaico de Campeche, México. Contactos, 63, 5-14.

Pielou, E. C. (1966). The measurement of diversity in different types of biological collections. Journal of Theoretical Biology, 13, 131-144.

Pombo, L., Elliott, M., \& Rebelo, J. E. (2005). Environmental influences on fish assemblage distribution of an estuarine coastal lagoon, Ria de Aveiro (Portugal). Scientia Marina, 69(1), 143-159.

Reséndez-Medina, A., \& Kobelkowsky-Díaz, A. (1991). Ictiofauna de los sistemas lagunares costeros del Golfo de México, México. Universidad y Ciencia, $8,91-110$. 
Shannon, E. C., \& Wiener, W. (1963). The Mathematical Theory of Communication. Illinois: University of Illinois.

Snedden, G. A., Cable, J. E., \& Kjerfve, B. (2013). Estuarine geomorphology and coastal hidrology. In J. W. Day, B. C. Crump, W. M. Kemp, \& A. Yáñez-Arancibia (Eds.), Estuarine ecology (pp. 19-38). Nueva York: Wiley-Sons.

Ter Braak, C. J. F., \& Verdonschot, P. F. M. (1995). Canonical correspondence analysis and related multivariate methods in aquatic ecology. Aquatic Science, 57, 255-289.

Utrera-López, M. E., \& Moreno-Casasola, P. (2008). Mangrove litter dynamics in La Mancha lagoon, Veracruz,
México. Wetlands Ecology and Management, 16, 11-22.

Velázquez-Velázquez, E., Vega-Cendejas, M. E., \& Navarro-Alberto, J. (2008). Spatial and temporal variation of fish assemblage in a coastal lagoon of the Biosphere Reserve La Encrucijada, Chiapas, Mexico. Revista de Biología Tropical, 56, 557-574.

Yáñez-Arancibia, A., \& Lara-Domínguez, A. L. (1988). Ecology of three sea catfishes (Ariidae) in a tropical coastal ecosystem southern Gulf of Mexico. Marine Ecology Progress Series, 49, 215-230.

Zar, J. H. (1999). Biostatistical Analysis. Nueva Jersey: Prentice-Hall. 
